\title{
INTERRELACIONES ENTRE LA EDUCACIÓN FORMAL Y LA EDUCACIÓN NO FORMAL EN LA TRANSMISIÓN DE VALORES EUROPEOS
}

\author{
INTERSEÇÕES DE EDUCAÇÃO FORMAL E EDUCAÇÃO NÃO-FORMAL EM \\ TRANSMISSÃO DE VALORES EUROPEU \\ INTERRELATIONSHIPS BETWEEN FORMAL EDUCATION AND NON FORMAL \\ EDUCATION IN THE TRANSMISIÓN OF EUROPEAN VALUES
}

\begin{abstract}
Alfonso Conde Lacárcel (alfconl8@ugr.es) *
Mohammed El Homrani (mohammed@ugr.es) *
\end{abstract}

\section{RESUMEN:}

A través de la metodología de talleres participativos de trabajo se ha pretendido mostrar la interrelación que hay entre los sistemas educativos formales y la educación no formal en el desarrollo de valores democráticos y la educación ciudadana en el contexto europeo; en especial en las Organizaciones No Gubernamentales (ONG) que complementan los distintos proyectos y programas en Europa. Fue llevado a cabo dentro de un Intensive Programme ERASMUS (IP-ERASMUS). El objetivo principal de este workshop consistió en hacer conscientes al profesorado y alumnado participante de la labor que realizan estos agentes externos, así como desarrollar valores de empatía, colaboración y compromiso. Al final del mismo se llevó a cabo una evaluación de la labor desarrollada con el fin de comprobar el grado de consecución de nuestros objetivos y en qué aspectos el auditorio asistente se centra más a la hora de participar en este tipo de actividades. Los resultados obtenidos se centran en la aplicabilidad práctica de la temática desarrollada, su grado de profundidad y la capacidad del disertante para captar la atención del auditorio, así como su experiencia en la materia.

Palabras-clave: educación ciudadana, talleres, educación superior.

\section{RESUMO:}

Através da metodologia de oficina, tentamos mostrar a inter-relação entre os sistemas de educação formal e educação não formal no desenvolvimento de valores democráticos e a educação para a cidadania no contexto europeu; especialmente ao nível das Organizações Não-Governamentais (ONG), como complementar a diversos projetos e programas Europeus. Esta ideia foi conduzida no decorrer de um Intensive Programme ERASMUS (IP-ERASMUS). O principal objetivo passava por fazer com que os participantes tivessem consciência do trabalho que é realizado pelos agentes externos que pretendem desenvolver os valores da empatia, cooperação e compromisso. No final do mesmo foi realizada uma avaliação do trabalho, a fim de avaliar o grau de realização dos nossos objetivos e de que forma os participantes ficam mais focados quando participam nestas actividades.

Palavras-chave: Educação para a Cidadania, seminários, ensino superior. 


\title{
Eqitania \\ s c i e $\cap$ c i a
}

\begin{abstract}
:
Through the methodology of participatory workshops we have tried to show the interrelationship between formal education systems and non-formal education in the development of democratic values and citizenship education in the European context; especially Non-Governmental Organizations (NGO) complement various projects and programs in Europe. It was performed in the framework of Intensive Program ERASMUS (IP-ERASMUS). The main objective of this workshop was to make faculty and students aware participant in the ongoing work of these external agents, and develop values of empathy, cooperation and commitment. At the end of it took place an evaluation of the work done in order to verify the degree of achievement of our goals and in what respects audience is more focused assistant when participating in these activities. The results focus on the practical applicability of the developed topic, level of depth and the ability of the speaker to capture the audience's attention as well as their experience in the field.
\end{abstract}

Keywords: citizenship education, workshop, high education.

*Universidade de Granada

Submitted: 11th November 2014

Accepted: 5th December 2014 


\section{Egitania \\ $s$ c i e $\Omega$ c i a}

\section{INTRODUCCIÓN: CIUDADANÍA EUROPEA Y EDUCACIÓN}

El desarrollo de la ciudadanía europea ha sido y es una preocupación constante percibida desde los distintos sistemas educativos de los 25 países que integran la Unión Europea (Pepín, 2007); no solo como una manera de cohesionar y favorecer los valores democráticos de inclusión social y de participación (Coster, Borodankova, De Almeida \& Paolini, 2012), sino como un elemento de crecimiento económico y de desarrollo. Sin embargo, la aplicación y priorización por parte de los distintos estados miembros y la asignación de recursos no han sido siempre los más adecuados, debido a las distintas apreciaciones de éstos sobre los aspectos considerados prioritarios a nivel educativo.

De acuerdo con Karlsen (2002) citado por Agirdag, Huyst y Van Houtte (2012, p. 202): "La Unión Europea ha gastado millones de euros en educación, formación y programas para la juventud en un intento de animar a los jóvenes a entrar en el mercado profesional europeo y prepararlos para sus roles como ciudadanos europeos".

En 2012, el Informe "Citizenship Education in Europe" publicado por Eurídice nos recordaba que la Educación para la Ciudadanía Democrática se ha incorporado en todas las etapas de los sistemas educativos europeos. "Por su parte, los contenidos de derechos humanos están presentes en los currículos de más de 20 países europeos" (Coster, Borodankova, Coutinho y Paolini 2012 citados por Fundación Cides, 2013, parr.3).

Sin embargo, ¿a qué podemos llamar "identidad europea ciudadana" y cómo podemos fomentarla? Según Patrutui (2013) son: "Un conjunto de valores compartidos por todos los ciudadanos de la Unión Europea" (párr. 3).

Las principales líneas de investigación centradas en la educación ciudadana y la identidad europea, han estado referidas principalmente a factores como el género, la edad o el nivel socioeconómico (Medrano y Gutiérrez, 2001; Rise, 2005; Bruter, 2008). Por contra, otros factores como son la religión, la etnia o el contexto escolar apenas han sido tenidos en cuenta, demostrándose, junto con variables individuales, como influyentes a la hora de fomentar esa identidad (Agirdag, Huyst y Van Houtte, 2011).

Será a partir de las competencias sociales y cívicas desde las que partamos para construir una ciudadanía europea que genere este sentido de pertenencia y compromiso por parte de los ciudadanos europeos, dentro del curriculum desarrollado por los distintos países. Estas competencias ciudadanas: "Preparan a las personas para participar plenamente en la vida cívica, basándose en el conocimiento de conceptos sociales y políticos, y sus estructuras, y un compromiso a la participación activa y democrática (EU, 2007, p.9).

Pero no siempre es posible desarrollar adecuadamente este currículo en las aulas, por lo que un elemento decisivo interrelacionado con los sistemas educativos formales creemos que es la educación no formal, como agente complementario a estas políticas que desarrollan valores sociales comunes, de cohesión y lucha contra la exclusión (Colom, 2005). Siguiendo a Domínguez, Lamata y Baraibar (2003 pp.53-54), el ámbito sociocomunitario intenta establecer una interrelación con los sistemas formales educativos con el fin de mantener la calidad educativa de sus acciones. 


\section{Egitania \\ s c i e $\Omega$ c i a}

Aspectos como el pensamiento crítico; la participación ciudadana; y la justicia social están muy presentes en el desarrollo de actividades formativas y educativas por parte de estos agentes no formales. De esta manera, se posibilita el refuerzo en contextos positivos y cercanos, de la adquisición y transmisión de valores europeos y participación ciudadana.

Hoy día, la dificultad para afrontar los nuevos retos derivados de la globalización hacen necesario redoblar los esfuerzos para ser capaces de responder de manera coordinada con una educación de calidad desde los distintos agentes sociales (Escobar, 2010). Es por ello que partimos en el desarrollo de este taller de trabajo, de la necesidad de complementar los distintos proyectos y programas que se vienen realizando desde los sistemas educativos formales, con la ayuda de las organizaciones no gubernamentales (Ongs), organizaciones no lucrativas (Onls) y asociaciones que trabajan en distintos contextos y con grupos diversos étnica, cultural, religiosa o económicamente.

\section{METODOLOGÍA DE UN TALLER}

Desde nuestro deseo de realizar una práctica educativa crítica y comprometida con la realidad, este taller ha partido de una planificación estratégica orientada a la presentación de una perspectiva diferente en la adquisición de valores; en este caso de participación ciudadana.

"El taller constituye: a) un dispositivo de trabajo con y en grupos; [ I es limitado en el tiempo y se realiza con determinados objetivos particulares, permitiendo la activación de un proceso pedagógico sustentado en la integración de teoría y práctica, el protagonismo de los participantes, el diálogo de saberes, y la producción colectiva de aprendizajes, operando una transformación en las personas participantes y en la situación de partida (Cano, 2012; p.33)."

Los objetivos que nos propusimos respecto a la temática planteada fueron:

_Presentar la educación ciudadana en Europa desde la complementariedad entre los sistemas educativos formales y no formales.

_Destacar la labor realizada por los agentes sociales mencionados (ONGs, ONLs).

_Desarrollar valores de empatía, colaboración y compromiso entre instituciones, organizaciones, grupos.

En este Intensive Programme Erasmus participaron 9 universidades de la Unión Europea (España, Alemania, Grecia, Letonia, Lituania, Rumanía, República Checa, Dinamarca y Turquía). Fue implementado con un grupo compuesto por 85 participantes (24 profesores y 61 estudiantes; estos últimos con edades comprendidas entre los 18 y 25 años).

El programa se desarrolló en una sesión de mañana de 3 horas de duración divididas en: hora y media de conferencia (Lectura) en la que se presentaban nuestras aportaciones a la temática con debate posterior y confrontación de ideas; y realización posterior de trabajo 


\section{Egitania \\ $\mathrm{s}$ c i e $\Omega$ c i a}

grupal en pequeños grupos de 6-8 personas con una dinámica de intervención social educativa relacionada con todo lo expuesto, de una hora y media de duración (Taller) con presentación de las conclusiones y evaluación final mediante un cuestionario de satisfacción realizado al final de la jornada.

Este instrumento fue contestado finalmente por 62 encuestados del total de personas participantes (72\% debido al carácter voluntario); 23 no fueron contestados (28\%). El análisis de los resultados en base a estos datos creemos que nos aporta una base para apreciar el desarrollo del trabajo realizado en su conjunto.

El cuestionario de satisfacción posee una fiabilidad Alfa de Cronbach de 0'707 y se compone de una escala Lickert de 1-5, de menor a mayor grado de concordancia entre los distintos aspectos tratados en el desarrollo del Workshop. Dividido en dos partes, la primera hace referencia a los contenidos: su elección, profundidad y aplicabilidad. En la segunda parte del cuestionario, abordamos aspectos relacionados con el disertante: nivel de conocimientos, aplicación de su experiencia profesional al conjunto del Workshop, así como el grado de profundidad en la Lectura.

Se incluyen igualmente dos apartados de respuesta abierta, con el fin de complementar los datos analizados con información relevante, que fueron analizadas a través de las técnicas del discurso biográfico- narrativo y en base a las dimensiones propuestas para la parte cualitativa del cuestionario de satisfacción. Pasamos a desarrollarlos a continuación.

\section{RESULTADOS}

Los resultados obtenidos en los distintos apartados del cuestionario, a pesar de ser positivos en su mayoría y constatar el trabajo realizado, deben ser mejorados a tenor de las respuestas encontradas en las preguntas abiertas.

Los participantes en la Lectura y el Taller, demandaron la necesidad de incluir más información referida a:

Las distintas formas de implementar y llevar a cabo la educación no formal en la práctica.

¿Cómo conseguir desarrollar en la educación formal la ciudadanía e identidad europea?

El grado de aplicabilidad real de este tipo de acciones (complementariedad entre educación formal y no formal).

En las gráficas mostradas a continuación presentamos las frecuencias y los porcentajes de respuesta asociados de manera que permitan una mejor comprensión de los datos analizados. Comenzamos con la elección de la temática tratada. En este caso, y cómo podemos ver en la Figura 1, un 66,1\% de los participantes (41 participantes) consideraron "buena" la elección de la temática y un 32,3\% "excelente" (20 participantes). 


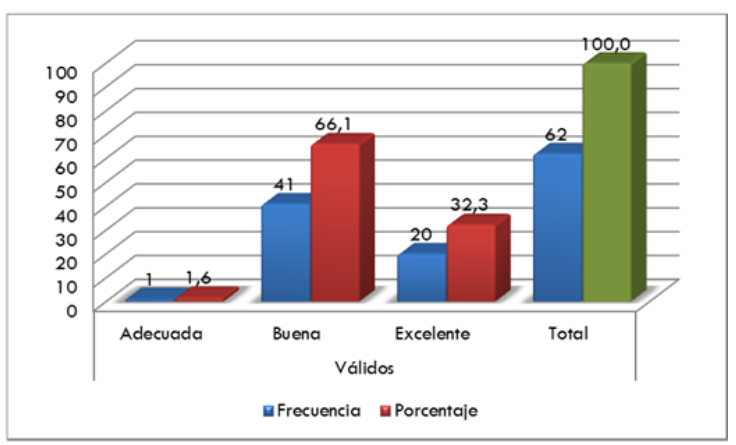

Figura 1: Elección de los temas tratados

El grado de profundidad, teniendo en cuenta los tiempos marcados fue percibida en un 59,7\% como "buena" (37 participantes); y un 17,7\% "excelente" (11 participantes) como podemos ver en la Figura 2. Este punto contrasta en las respuestas abiertas con algunos de los participantes, que tuvieron una percepción de dificultad para seguir el Taller alegando un exceso de información. (Respuesta de uno de los encuestados: "difficult to understand during the Lecture, but during the Workshop very easy").

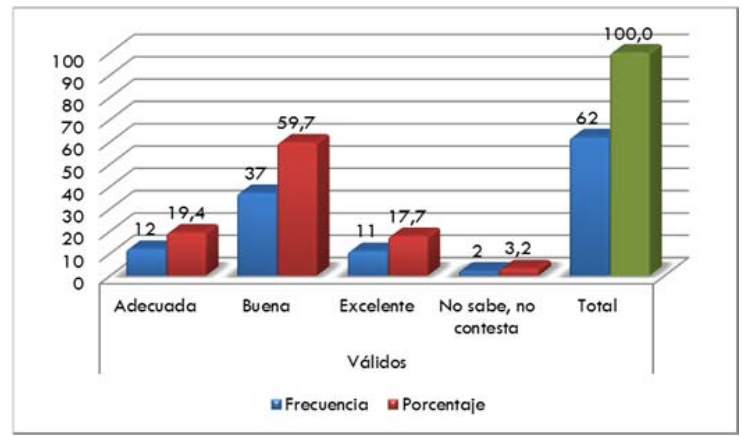

Figura 2: Grado de profundidad en el desarrollo de la temática

Respecto al grado de aplicabilidad a su área profesional, los resultados en la Figura 3, se diversifican entre un 54,8\% considerada "buena" (34 participantes); un 32,3\% "excelente" (20 participantes) y un 11,3\% "adecuada" (7 participantes).

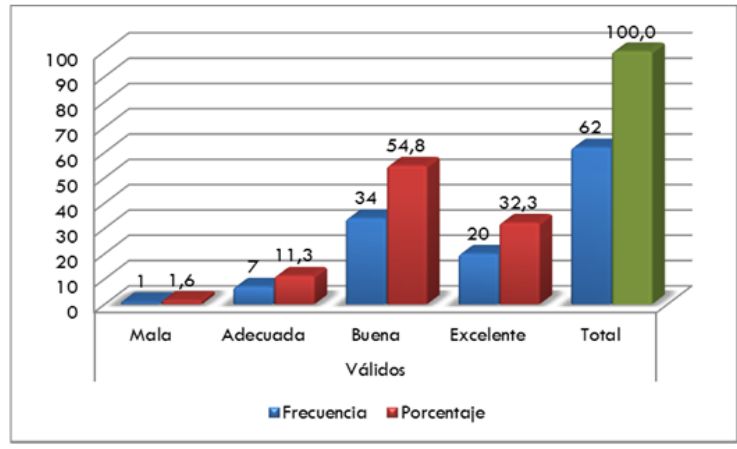

Figura 3: Grado de aplicabilidad a su área profesional 


\section{Eqitania}

s c i e $\Omega$ c i a

En la Figura 4, en respuesta al grado de utilidad para los participantes del Taller, nos encontramos con que a pesar, de las cuestiones planteadas respecto a la necesidad de cómo implementar la educación no formal en la práctica y el grado de aplicabilidad real, es considerado por un 83,9\% como "muy útil" (52 participantes). Solamente un 9,7\% lo consideró de "poca utilidad" (6 participantes).

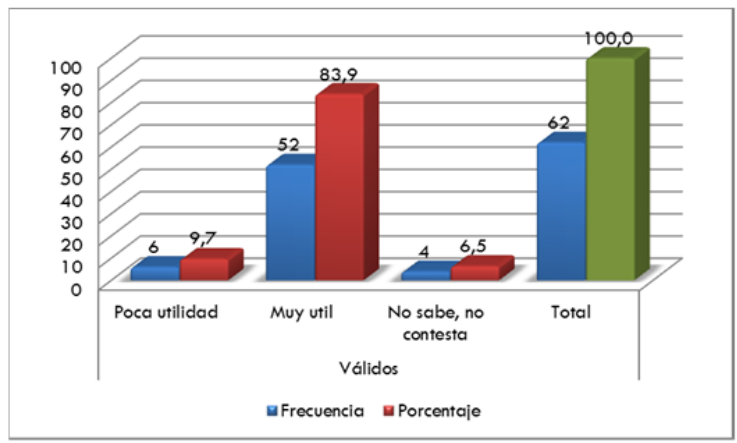

Figura 4: Grado de utilidad del Workshop para los participantes

Pasamos ahora a analizar las cualidades y desempeño del disertante, percibidas por los encuestados. En general, podemos decir que éste fue percibido como una persona profesional, con los recursos adecuados para llevar a cabo el Workshop (cabe decir que fue apoyado técnica y personalmente por compañeros y estudiantes en colaboración).

En la Figura 5 vemos que un 58\% consideró su nivel de conocimientos como "bueno" (36 participantes) y un 40\% de los encuestados lo percibieron como "excelente" (25 participantes).

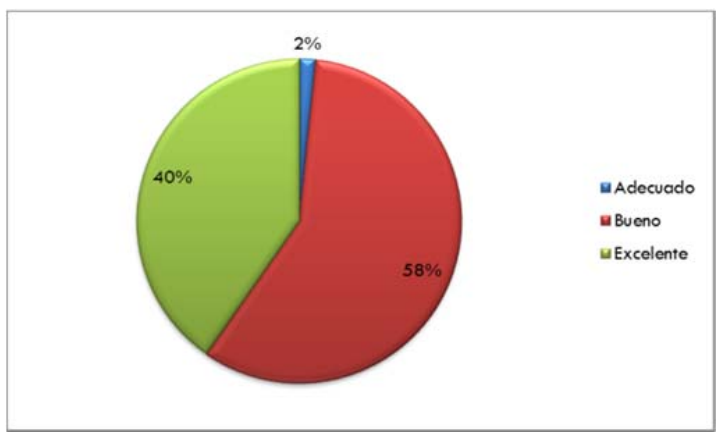

Figura 5: Nivel de conocimientos del disertante

La percepción general del auditorio respecto a su experiencia profesional y la aplicación al Taller, como vemos en la Figura 6, es considerada en un 61\% como "buena" (38 participantes), seguida de un 26\% "excelente" (16 participantes) y un 10\% "adecuada" (6 participantes) 


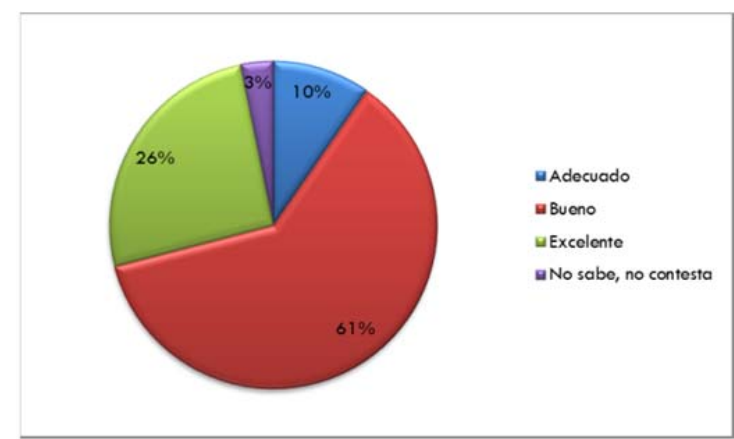

Figura 6: Grado de aplicación de la experiencia profesional del disertante a la conferencia /exposición / Workshop

Por otra parte, y a pesar de ser un Taller desarrollado íntegramente en inglés por un conferenciante hispanoparlante en la Figura 7 vemos que un 58\% consideró su velocidad de desarrollo "buena" (36 participantes); seguido de un 24\% que la consideraron "excelente" (15 participantes) y un 16\% "adecuada" (10 participantes).

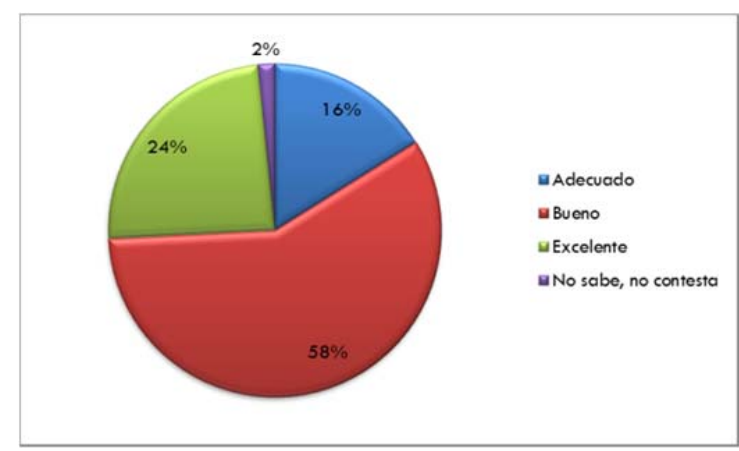

Figura 7: Velocidad de desarrollo de la conferencia / exposición / Workshop

"Actually, it was quite good knowing that it was first time for audience full of foreigners, giving lecture in English" (Respuesta dada por uno de los encuestados).

Por ultimo en la Figura 8, podemos ver que los participantes en el conjunto del Taller con Lectura, el grado de profundidad desarrollado es considerado "bueno" en un 53\% (33 participantes); un 29\% lo considera "excelente" (18 participantes) y un 15\% "adecuado" (9 participantes).

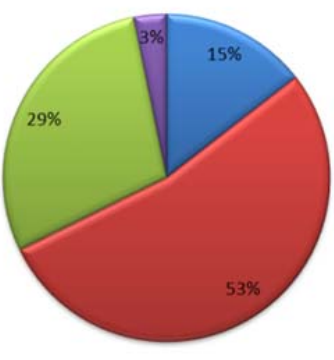

घAdecuado

a Bueno

$\square$ Excelente

a No sabe, no contesta 


\section{Egitania \\ s c i e $\Omega$ c i a}

Figura 8: Grado de profundidad de la conferencia / exposición / Workshop

\section{CONCLUSIONES}

Como conclusión, podemos decir que el Taller desarrollado fue percibido como positivo en todas sus facetas, tanto para los participantes como para el equipo organizador de éste. La temática fue considerada como interesante y necesaria en relación a las cuestiones planteadas, contribuyendo al trabajo desarrollado desde el Intensive Programme Erasmus en colaboración con el resto de universidades europeas participantes.

En relación con los objetivos propuestos, y a tenor de los resultados obtenidos en la evaluación del mismo, creemos haberlos alcanzado. En especial, el desarrollo de valores de empatía, colaboración y compromiso entre los asistentes.

Desde el punto de vista organizativo y de implementación del Taller, es preciso por nuestra parte, complementar los contenidos aportados al auditorio con información referida a las distintas metodologías utilizadas en la educación no formal para desarrollar sus actividades con los distintos grupos y realidades. Esta fue una demanda realizada por la mayoría de los asistentes.

Otro aspecto planteado también como futura línea de trabajo en referencia a la educación ciudadana y los valores europeos al llevar a cabo un análisis para futuros Talleres, reside en la concepción de las personas que integran los distintos países de la Unión Europea sobre lo que realmente significa y conlleva la idea de identidad europea.

\section{BIBLIOGRAFIA}

Agirdag, O., Van Houtte, M. \& Van Avermaet, M. (2011) 'Ethnic School Context and the National and Sub-national IDENTIFICATIONS OF PUPILS'. ETHNIC AND RACIAL StUdies, 32, (2), 357-78.

Agirdag, O., Huyst, P., \& Van Houtte, M. (2012). Determinants of the Formation of a European IDentity among ChildRen: IndiVIDUAL-AND SCHOOL-LeVel INFlUenCES. JCMS: JOURNAL OF COMMON MARKet StUdies, 50, (2), 198-213.

Bruter, M. (2008) 'Legitimacy, Euro scepticism and Identity in the European Union: Problems of Measurement, Modelling and Paradoxical Patterns of InfluencE'. Journal OF CONTEMPORARy EuROPEAN RESEARCH, 4, (4), 273-85.

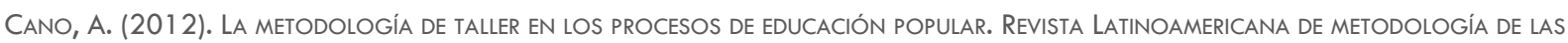
CIENCIAS SOCIALES, 2 (2), 22-51. EN MEMORIA ACADÉMICA.

RECUPERADO DE HTTP://WWW.MEMORIA.FAHCE.UNLP.EDU.AR/ART_REVISTAS/PR.5653/PR.5653.PDF

COlOM, A. (2005). CONTINUIDAD Y COMPLEMENTARIEDAD ENTRE LA EDUCACIÓN FORMAL Y NO FORMAL. REVISTA DE EdUCACIÓN (338), 9-22.

Domínguez, R., LAMATA, R., \& Baraibar, J.M. (2003). LA CONSTRUCCIÓN DE PROCESOS FORMATIVOS EN EDUCACIÓN NO FORMAL. MADRID: NARCEA.

De Coster, I., Borodankova, O., De Almeida Coutinho, A. S., \& Paolini, G. (2012). Citizenship education in Europe. Education, Audiovisual and Culture ExeCutive Agency, European Commission. AVallable from EU BoOKSHOP.

ESCOBAR, R.A. (2010). LAS ONG COMO ORGANIZACIONES SOCIALES Y AGENTES DE TRANSFORMACIÓN DE LA REALIDAD: DESARROLLO HISTÓRICO, EVOLUCIÓN Y CLASIFICACIÓN. DIÁlOgOS de SABERES: INVESTIGACIONES Y CIENCIAS SOCIALES (32), 121 -131. 


\section{Eqitania \\ s c i e $\Omega$ c i a}

European Commission. (2007). The Key Competences for Lifelong learning - A European Framework. LuXembourg: Office for OfFicial Publications OF the European Communities. Recuperado el 30/06/2014 DE HTTP://WWW.BRITISHCOUNCIL.ORG/SITES/BRITISHCOUNCIL.UK2/FILES/YOUTH-IN-ACTION-KEYCOMP-EN.PDF

Fundación Cives (2013). Memorándum al Consejo de Europa sobre el proyecto del Gobierno de suprimir la Educación para la

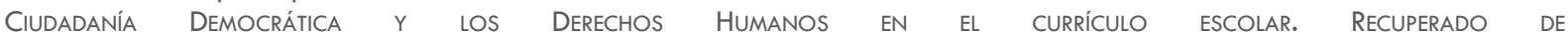
HTTP://WWW.FUNDACIONCIVES.ORG/COMUNICACION/NOTICIAS/MEMORANDUM-AL-CONSEJO-DE-EUROPA-SOBRE-EL-PROYECTO-DEL-GOBIERNODE-SUPRIMIR-LA-EDUCACION-PARA-LA-CIUDADANIA-DEMOCRATICA-Y-LOS-DERECHOS-HUMANOS-EN-EL-CURRICULO-ESCOLAR/

Medrano, J.D. \& GutiérRez, P. (2001) 'Nested Identities: National and European Identity in Spain'. Ethnic and Racial Studies, 24,(5), 753-78.

Patrutiu, L. (2013). Europe and Europeans-Questions of identity. One. Recuperado el 14/10/2013 de htTp://OneEUROPE.INFO/EUROPE-AND-EUROPEANS-QUESTIONS-OF-IDENTITY

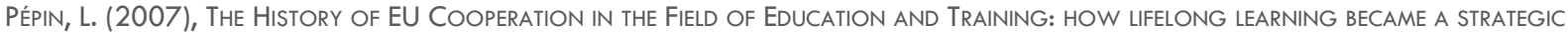
OBJECTIVE. EUROPEAN JOURNAL OF EDUCATION, (42), 121-132.

Risse, T. (2005) 'NeOfunctionalism, European Identity and the PuzZles of European Integration'. Journal Of European Public Policy $12,(2), 291-309$. 